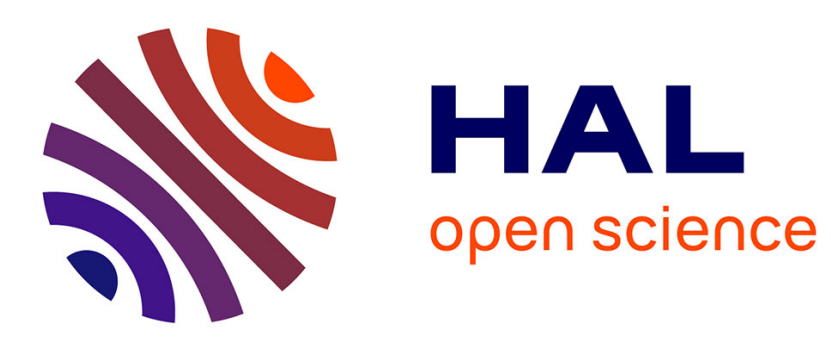

\title{
La tradition des ouvres de Jean Chrysostome, entre transmission et transformation
}

\author{
Guillaume Bady
}

\section{To cite this version:}

Guillaume Bady. La tradition des œuvres de Jean Chrysostome, entre transmission et transformation. Revue des études byzantines, 2010, 68, pp.149-163. 10.3406/rebyz.2010.3066 . halshs-00537414

\section{HAL Id: halshs-00537414 \\ https://shs.hal.science/halshs-00537414}

Submitted on 27 Jan 2020

HAL is a multi-disciplinary open access archive for the deposit and dissemination of scientific research documents, whether they are published or not. The documents may come from teaching and research institutions in France or abroad, or from public or private research centers.
L'archive ouverte pluridisciplinaire HAL, est destinée au dépôt et à la diffusion de documents scientifiques de niveau recherche, publiés ou non, émanant des établissements d'enseignement et de recherche français ou étrangers, des laboratoires publics ou privés.

\section{(1)(1) $\$(0)$}

Distributed under a Creative Commons Attribution - NonCommercial - ShareAlikel 4.0 


\section{La tradition des oeuvres de Jean Chrysostome, entre transmission et transformation \\ Guillaume Bady}

\section{Résumé}

Ce que l'on sait de Chrysostome dépend de ce que transmettent les manuscrits, les textes édités et les études modernes. Or, étant donné l'impossibilité d'aboutir à des éditions critiques définitives et de retrouver un original utopique, la déconstruction critique de l'édifice chrysostomien mène en fait à revenir à ce que les traditions les plus anciennes ont d'authentique et à reconnaître leur rôle propre. Texte, entourage du texte, séries de textes et textes dérivés : la tradition transforme l'oeuvre chrysostomienne en même temps qu'elle la transmet et la trahit.

\section{Abstract}

All that we know about Chrysostom comes from what has been transmitted by the manuscripts, edited texts and modern studies. But given the impossibility of producing definitive editions or of reconstructing a perfect original, critical deconstruction of the Chrysostomian edifice leads us back to that which is authentic in the most ancient traditions and the recognition of their role : the text itself, that which accompanies it, series of texts and derived texts. Tradition transforms the Chrysostomian oeuvre, at once transmittting and distorting it.

\section{Citer ce document / Cite this document :}

Bady Guillaume. La tradition des oeuvres de Jean Chrysostome, entre transmission et transformation. In: Revue des études byzantines, tome 68, 2010. pp. 149-163;

doi : 10.3406/rebyz.2010.3066

http://www.persee.fr/doc/rebyz_0766-5598_2010_num_68_1_3066

Document généré le 24/01/2017 


\title{
LA TRADITION DES CEUVRES DE JEAN CHRYSOSTOME, ENTRE TRANSMISSION ET TRANSFORMATION
}

\author{
Guillaume BADY
}

La figure traditionnelle de Jean Chrysostome est aujourd'hui réexaminée depuis sa base: l'heure est à la déconstruction ${ }^{1}$. W. Mayer parle même de «démolition» concernant la chronologie des œuvres de Jean Chrysostome et l'unité de certaines séries homilétiques, telles qu'avaient pu les établir plusieurs siècles d'érudition ${ }^{2}$. $\mathrm{Si}$, par «Chrysostome», en effet, on entend la figure «canonisée» par Fronton du Duc, H. Savile, B. de Montfaucon, J.-P. Migne, Ch. Baur et A.-M. Malingrey, il semble nécessaire de dessiner quelques limites et perspectives de notre épistémologie, et de reconnaître le rôle de la tradition - celle des manuscrits comme celle des éditions modernes - dans la connaissance que nous avons de Jean Bouche d'Or. Car en réalité, celle-ci repose en grande partie sur les éditions des $17^{\mathrm{e}}$ et $18^{\mathrm{e}}$ siècles, notamment celle de Montfaucon reproduite dans la Patrologie grecque.

L'un des aspects de cette dépendance est la mise en ordre des œuvres complètes de l'Antiochien - un ordre tout à fait méritoire, permettant de s'orienter dans cette «forêt» d'écrits. Le plan implicite qui y préside peut être sommairement décrit en 3 ou en 8 parties, en excluant les dubia et spuria:

I. Euvres diverses:

1. Euvres ascétiques (moines, vierges, veuves, prêtres): $P G$ 47, 277 - 48, 700

2. Euvres polémiques: $P G 48,701-942$

3. Euvres liées au cycle liturgique, du carême à la Pentecôte: $P G 48,953$ $-50,470$

4. Panégyriques: $P G$ 50, 473-712

5. Euvres pastorales et exégétiques: $P G 51,17-388$

6. Euvres liées aux circonstances historiques (399-403) : PG 52, 391-748

II. 7. Séries d'homélies exégétiques: $P G$ 53, $21-63,236$

III.8. Autres œuvres : $P G$ 63, $467-64,1037$.

1. Cet article reprend en partie une communication faite le 24 novembre 2007, lors du Colloque patristique sur saint Jean Chrysostome, à l'Institut de Théologie Orthodoxe SaintSerge, Paris.

2. W. MAYER, Biography and Chronology, communication présentée en collaboration avec M. Walraff au colloque Chrysostomika II, Augustinianum, Rome, le 8 novembre 2007. 
Outre le relatif désordre, dû parfois aux aléas de l'édition d'opera omnia, cette disposition actuelle a plusieurs défauts, qui remontent en partie à la tradition manuscrite. D'une part, l'appartenance artificielle de telle homélie à telle série, ou au contraire un isolement indu, ou encore un titre mal choisi ne facilitent pas la juste compréhension de l'œuvre. D'autre part, comme en témoignent la Clauis Patrum Graecorum et son supplément ${ }^{3}$, et sans parler des dubia et spuria inclus dans la Patrologie (CPG 4500-4713) ou non (CPG 4715-5197), le corpus chrysostomien est plus vaste, car il y a des œuvres éditées après la Patrologie ( $C P G$ 4455-4472 notamment), ainsi que des fragments (CPG 4495).

Qui plus est, il est aisé d'oublier que notre connaissance de l'œuvre chrysostomienne est nécessairement partielle, étant donné la portion importante de ses écrits qui a disparu: des homélies de Chrysostome, il ne reste que $20 \%$ si l'on ne prend en compte que les homélies réputées authentiques, conservées au nombre de 760 sur un total que l'on peut estimer à 4000 . Car si un orateur comparable comme Augustin, sur une période de 40 ans, a prêché quelque 8000 fois ${ }^{4}$, Chrysostome a dû le faire 4000 fois sur 20 ans, y compris la période d'exil où l'on sait qu'il a continué d'une façon ou d'une autre à se faire entendre. Toute analyse se doit dès lors de prendre en compte l'effet d'optique éventuellement grossissant ou réducteur opéré par le choix, même large, des textes transmis.

Or, par un paradoxe qui n'est qu'apparent, puisqu'il s'agit en réalité d'un aboutissement logique, la déconstruction en cours exige la construction de nouvelles fondations, à savoir des éditions critiques fiables: «Tant qu'on ne peut travailler avec des textes scientifiquement édités et authentifiés, toute recherche sur la théologie, l'éloquence, la vie ou l'influence de Chrysostome demeure conditionnelle ${ }^{5}$.

Le besoin d'éditions critiques que W. Mayer exprimait ainsi en 2004, impliquant une double nécessité, celle de déconstruire la tradition et d'y revenir, semble plus urgent que jamais ${ }^{6}$. Bien sûr, depuis les années 1960, la Section grecque de l'IRHT et ses collaborateurs contribuent grandement à une meilleure connaissance de la tradition manuscrite des œuvres chrysostomiennes. Le Repertorium pseudochrysostomicum de J. de Aldama paru en 1966 et les volumes des Codices Chrysostomici Graeci $^{7}$ sont très utiles : non

3. M. Geerard, Clauis Patrum Graecorum, II, Turnhout 1974, p. 491-672; M. Geerard et J. Noret, Clauis Patrum Graecorum, Supplementum, Turnhout 1998, p. 264-347.

4. P. Allen, It's in the post: techniques and difficulties of letter-writing with regard to the letters of Augustine, dans Proceedings 2005 of the Australian Academy of the Humanities, Canberra 2006, p. 111-129, spéc. p. 121.

5. W. Mayer, Progress in the Field of Chrysostom Studies (1984-2004), dans Giovanni Crisostomo. Oriente e Occidente tra IV e v secolo. XXXIII Incontro di studiosi dell'antichità cristiana (Roma, 6-8 maggio 2004), I, Rome 2005, p. 9-35, ici p. 17 (je traduis). W. Mayer fournit une bibliographie précieuse des travaux en Occident: à propos des éditions critiques récentes, voir p. 11-18.

6. M. Aubineau disait déjà en 1968 qu'une enquête «urgente» s'imposait: Une enquête dans les manuscrits chrysostomiens: opportunité, difficultés, premier bilan, Revue d'histoire ecclésiastique 63, 1968, p. 5-26, spéc. p. 7.

7. Le projet a été formé à l'initiative de Robert E. Carter dès 1956. 
seulement pour obtenir des informations concernant tel ou tel manuscrit, mais aussi pour avoir une idée, même partielle, de la façon dont un texte est transmis en général dans la tradition grecque ${ }^{8}$.

Néanmoins, la production d'éditions critiques, malgré le dévouement sans bornes, et de plus en plus méthodique, de spécialistes comme A.-M. Malingrey, est restée limitée. La seule idée d'accomplir ce qui pourtant a déjà été fait à trois reprises par quelques individus, c'est-à-dire une édition des œuvres complètes, est tout bonnement utopique, même dans un cadre institutionnel et éditorial comme celui des Sources Chrétiennes. Il est peu vraisemblable, en effet, que la collection puisse relever le défi de publier quelque 1500 écrits, qui occupent plus de 10000 colonnes grecques de Migne ${ }^{9}$ et qui représenteraient plus de 100 volumes de Sources Chrétiennes ${ }^{10}$; des écrits qui eux-mêmes sont transmis par des milliers de manuscrits de par le monde ${ }^{11}$, dans des langues et des traditions très diverses. Les conditions socio-économiques de la recherche, l'état des connaissances, le nombre des documents virtuellement exploitables, la complexité grandissante des méthodes et des problématiques, les exigences scientifiques modernes en général rendent très improbable l'existence d'un nouveau Savile au $21^{\mathrm{e}}$ siècle.

Le premier problème réside donc dans l'immensité concrète de l'entreprise d'ecdotique, elle-même susceptible, qui plus est, de révisions infinies. Bien peu pourront donc décemment se permettre l'ambition d'une editio critica maior, se consolant tout de même à l'idée que beaucoup de progrès peuvent être faits en comparaison avec le texte de la Patrologie grecque; souvent, il est vrai, la montagne risque d'accoucher d'une souris, et le travail de fourmi pourrait dans certains cas apporter pour toute amélioration le changement d'un $\tau \varepsilon$ en $\delta \varepsilon^{\prime}$; le risque ne doit cependant pas être exagéré.

Un second problème tient au fait que le chercheur ne parviendra probablement jamais à remonter à «l'original», tout au mieux au $5^{\mathrm{e}}$ siècle par le biais du syriaque, mais déjà alors sans aucune garantie de parfaite authenticité, puisque la production de pseudochrysostomica a commencé du vivant même de Chrysostome. Une parfaite authenticité est d'ailleurs utopique: il convient

8. Il est prévu que la collection comprenne vingt volumes en tout; six ont paru à ce jour.

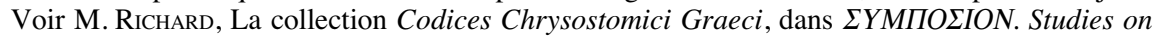
St. John Chrysostom (Analecta Blatadôn 18), éd. P. C. Christou, Thessalonique 1973, p. 91-96; P. Augustin, Le programme des Codices Chrysostomici Graeci (1956-2006). Un inventaire exhaustif des manuscrits chrysostomiens grecs, dans Le manuscrit dans tous ses états, cycle thématique 2005-2006 de l'IRHT (Ædilis, Actes, 12), éd. S. Fellous et ALII, http://aedilis.irht. cnrs.fr/manuscrit/chrysostome.htm.

9. L'estimation de M. Aubineau, Une enquête, cité n. 6, p. 6, englobe les pseudochrysostomica.

10. Seuls 20 d'entre eux sont publiés - ce qui fait tout de même de Jean Chrysostome, après Origène (40 et plus) et Tertullien (23), le $3^{\mathrm{e}}$ auteur de la Collection en nombre de volumes. On peut estimer à 20000 le nombre de pages de texte grec voulues pour cette Collection.

11. La base PINAKES, mise en ligne par la Section grecque de l'IRHT, permet d'estimer, toutes précautions prises, le nombre de manuscrits grecs de Jean Chrysostome à plus de 5000 , dont 1000 , par exemple, au Mont Athos, 800 dans le reste de la Grèce, un peu plus de 600 en Italie, un peu moins de 600 en France ou au Vatican. 
plutôt de parler de degrés d'authenticité ${ }^{12}$. Plus généralement arrivera-t-on à un état textuel du $9^{\mathrm{e}}$ ou du $10^{\mathrm{e}}$ siècle, tel qu'en témoignent souvent les plus anciens manuscrits ${ }^{13}$, datant de l'époque de la translittération massive des manuscrits onciaux en minuscules. Or cette étape décisive de la transmission des œuvres antiques s'accompagne d'une certaine normalisation des textes, qui pourrait bien se vérifier pour ceux de Jean Chrysostome et, ce faisant, fournir à notre besoin de nouvelles fondations le soutien d'une base assez solide.

Un tel constat suppose bien sûr que l'on renonce à l'authenticité originelle et que l'on reconnaisse à cette tradition ancienne le rôle qui est le sien: si imparfaite et inauthentique soit-elle, son statut semble épistémologiquement plus légitime que celui d'éditions modernes faites parfois sur les premiers manuscrits venus ${ }^{14}$. En tout état de cause, l'hypothèse de travail selon laquelle la tradition textuelle des $9^{\mathrm{e}}$ et $10^{\mathrm{e}}$ siècles a normalisé l'œuvre chrysostomienne ${ }^{15}$ n'empêche évidemment pas l'historien des textes de viser à travers elle l'origine, celle des $4^{\mathrm{e}}$ et $5^{\mathrm{e}}$ siècles, qu'elle doit plus ou moins fidèlement refléter. La Correspondance d'exil donne en tout cas l'exemple d'une tradition ancienne relativement homogène, qui permet d'améliorer le texte des éditions modernes faites en grande partie avec des recentiores; et de même le texte des 67 Homélies sur la Genèse, dont C. Crépey ${ }^{16}$ doit bientôt publier les dix premières aux Sources Chrétiennes, est susceptible de gagner un peu en antiquité autant qu'en fiabilité.

Beaucoup de questions resteront donc vraisemblablement sans réponse concernant la composition et la transmission des auvres de Chrysostome ${ }^{17}$.

12. Cf. W. MAYER, Biography and Chronology, cité n. 2.

13. Le plus ancien manuscrit de Chrysostome est un oncial, le Wolfenbüttel, Herzog August Bibliothek, Helmst. cod. 75a (début du $7^{\mathrm{e}} \mathrm{s}$.), contenant une partie des Homélies sur Matthieu. Voir notamment O. von HeInemann, Die Handschriften der Herzoglichen Bibliothek zu Wolfenbüttel. I, Die Helmstedter Hss., I, Wolfenbüttel 1884-1913, p. 77-78; W. MiLDE, Mittelalterliche Handschriften der Herzog-August-Bibliothek Wolfenbüttel, Frankfurt-am-Main 1972, p. 12; D. et J.HARLFInGER, J. A. M. SONDERKAMP et M. SicherL, Griechische Handschriften und Aldinen. Eine Ausstellung anläßlich der XV. Tagung der MommsenGesellschaft in der Herzog August Bibliothek Wolfenbüttel. Herzog August Bibliothek Wolfenbüttel, 16. Mai bis 29. Juni 1978, Wolfenbüttel 1978, p. 13-16.

14. Cf. M. Aubineau, Exploration dans une terra incognita de la littérature patristique et byzantine: les textes attribués à saint Jean Chrysostome, REG 78, 1965, p. XXXI-XXXIII, notamment p. XXXI.

15. A. Ehrhard parle même de la fin du $8^{\mathrm{e}}$ ou du début du $9^{\mathrm{e}}$ siècle, Überlieferung und Bestand der hagiographischen und homiletischen Literatur der griechischen Kirche von den Anfängen bis zum Ende des 16. Jahrhunderts (TU 50), I, Leipzig 1937, p. 5-35.

16. Auteur de la thèse: Jean Chrysostome: Homélies sur la Genèse (I-X; PG 53, 21-90). Introduction, traduction et notes, Univ. Paris IV-Sorbonne 2004.

17. Dans ma communication au Colloque scientifique international en commémoration du $1600^{\mathrm{e}}$ anniversaire de la mort de saint Jean Chrysostome organisé par le Patriarcat œcuménique de Constantinople du 14 au 17 septembre 2007, sous le titre: «St. John Chrysostom's Manuscript Tradition and his Publications: Some Basic Questions », j'ai énuméré dix d'entre elles, que j'aborderai rapidement un peu plus loin dans cet article:

1) Jean Chrysostome dictait-il ses écrits?

2) Improvisait-il ? 
En m'en tenant à la tradition grecque, et sans parler des traductions anciennes, je m'attacherai ici uniquement à cette question: quel est exactement le rôle de la tradition manuscrite dans la transmission et, plus précisément, dans la transformation des œuvres de Jean Chrysostome? Ma réponse consistera moins en une description qu'en une tentative de «modélisation» schématique, sous quatre rubriques: le texte, l'entourage du texte, les séries de textes, les textes dérivés ${ }^{18}$.

\section{1. - LE TEXTE}

Je n'ai sans doute pas besoin de m'attarder ici sur un phénomène aussi massif que structurel: les leçons divergentes, fautes ou variantes innombrables dans les manuscrits. Certaines variantes sont intentionnelles ${ }^{19}$, d'autres restent indécidables ou simplement indifférentes ${ }^{20}$.

Avant d'en venir aux problèmes généraux, certains cas particuliers méritent d'être mentionnés. Le premier est celui de l'harmonisation du texte biblique cité par l'auteur sur la vulgate byzantine: à un moment donné, un copiste a travaillé à partir de deux modèles, le manuscrit de Chrysostome à

3) Révisait-il ses textes ?

4) Quelle est la pertinence exacte, après la mort de Chrysostome, de la distinction entre discours prononcé et discours écrit?

5) En quoi exactement la tradition manuscrite a-t-elle modifié son œuvre?

6) Comment circulaient ses œuvres?

7) Comment les doubles recensions doivent-elles être interprétées ?

8) Dans quel contexte son œuvre a-t-elle été «publiée» à époque ancienne?

9) Dans quel contexte son œuvre a-t-elle été empêchée dans sa diffusion ou détruite?

10) Comment les doublons littéraires doivent-ils être interprétés?

18. À l'imitation du G. Genette de Palimpsestes et de Seuils, on pourrait employer ou inventer les mots suivants, respectivement: texte, péritexte, acoloutexte, apotexte. Mais ce genre de paratexte est-il vraiment utile?

19. Dans la lettre 112 adressée à Théodore de Mopsueste en des termes très favorables à ce

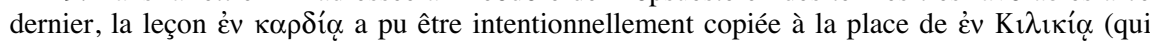
permettrait d'identifier immédiatement Théodore). C'est d'autant plus probable après les critiques formulées en 553 contre cette lettre par les Pères du concile de Constantinople II, devant lesquels les défenseurs des Trois-Chapitres, comme Facundus d'Hermiane, Défense des Trois Chapitres, VII, 7, 21-25, l'avaient produite; cf. le texte produit en réponse par les Pères conciliaires dans J. Straub, Acta conciliorum acumenicorum, IV, 1, Berlin 1971, p. 114. Même si le

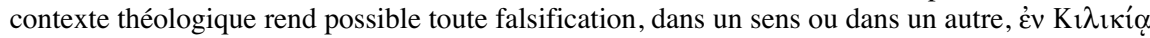
semble préférable comme lectio difficilior, mais elle n'est pas assez bien attestée aujourd'hui pour être retenue. Cf. G. BADY, Les traductions latines anciennes de Jean Chrysostome: motifs et paradoxes, dans Formation et transmission des collections textuelles de l'Antiquité tardive au Moyen Âge central (IVe-début XIII siècle) (Collection de l'École Française de Rome), éd. S. GioAnNi et B. Grévin, Rome 2008, p. 303-316, spéc. p. 315-316.

20. Ainsi dans les Lettres l'alternance, d'un manuscrit à l'autre, d'une lettre à l'autre ou même d'une ligne à l'autre, entre le singulier et le pluriel de la première personne: pour parler de lui-même, Jean Chrysostome employait à l'évidence les deux de façon concomitante. Voir, par exemple, C. CRÉPEY, L'influence du contexte sur le discours dans les Homélies sur la Genèse de Jean Chrysostome, dans Discorsi alla prova. Atti del Quinto Colloquio italo-francese, Napoli, 21-23 settembre 2006, éd. G. Abbamonte, L. Miletti et L. Spina, Naples 2009, p. 465-481, ici p. 475. 
copier et sa bible byzantine; lorsqu'il y avait une citation manifeste (généralement indiquée par une sorte de chevron en marge appelé $\delta \imath \pi \lambda \hat{\eta})$, il remplaçait le texte par le passage correspondant dans sa bible. En un temps qui n'est pas si éloigné, M.-É. Boismard aimait à rappeler l'heureuse intuition qu'il a eue un jour: constatant que le Commentaire sur Jean tel qu'il le lisait dans le tome 59 de la Patrologie ne comportait pas de variante majeure concernant l'évangile johannique, il a fini par consulter les manuscrits eux-mêmes et trouver des variantes par centaines, au point de l'amener à publier six volumes sur la présence, au sein du Commentaire, d'un évangile pré-johannique $^{21}$.

Le deuxième cas est celui de l'argumentum, ou író $q \varepsilon \sigma 1 \varsigma$, placé en tête d'une œuvre; six ou sept séries sur les lettres attribuées à Paul sont précédées d'un argumentum ${ }^{22}$. Alors que dans la littérature profane, l'argumentum est généralement le fait de la tradition et fait à ce titre partie du «paratexte», le cas de Chrysostome est particulier, dans la mesure où l'argument des homélies sur l'Épître aux Philippiens est considéré comme authentique et même comme la première des homélies. Le cas est encore plus net pour la série sur la Deuxième épître aux Thessaloniciens, où la première pièce porte chez

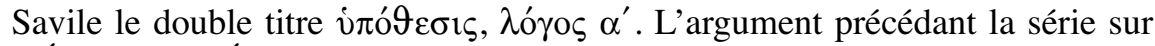
l'Épître aux Éphésiens est quant à lui très concis, comme celui sur la Première épître à Timothée. Soit il s'agit là d'une anomalie dans la transmission du texte (une homélie lacunaire), soit Chrysostome a vraiment fait précéder d'un argumentum écrit les séries d'homélies sur les épîtres pauliniennes, ce qui trahirait de sa part une responsabilité éditoriale par ailleurs peu manifeste $^{23}$. Ajout de la tradition, accident de transmission ou dessein proprement littéraire de l'auteur? Ou bien est-ce le résultat de la disparition, puis de la réapparition de la pratique de l'homélie au sein de la liturgie byzantine à la fin $\mathrm{du} 7^{\mathrm{e}}$ siècle, elle-même à peu près concomitante à la standardisation du cycle liturgique byzantin ${ }^{24}$ ? La présence d'un prologue dans le cas des commen-

21. M.-É. Boismard et A. Lamouille, Un évangile pré-johannique, vol. I, t. I, Paris 1993, p. 9.

22. Sur la Première épître aux Corinthiens, PG 61, 9-12; sur l'Épître aux Éphésiens, PG 62, 9-10; sur l'Épître aux Philippiens, PG 62, 177-182; sur la Première épître à Timothée, PG 62, 501-504; sur 1'Épître à Philémon, PG 62, 701-704; sur l'Épître aux Hébreux, PG 63, 9-14; le cas de la série sur la Deuxième épître aux Thessaloniciens, PG 62, 467-472, est ambigu; voir sur le problème des argumenta l'article de P. Allen et W. MAYER, Chrysostom and the preaching of homilies in series: A re-examination of the fifteen homilies In epistulam ad Philippenses (CPG 4432), Vigiliae Christianae 49, 1995, p. 270-289, spéc. p. 277-278 et 284.

23. À mon sens la présence d'un argumentum, qu'il résulte ou non d'une homélie, révèle davantage l'homogénéité d'une série comme objet éditorial délibéré que son hétérogénéité; P. Allen et W. MAYER, ibidem, p. 284, ont un avis inverse: «The homogeneity of the series is called into question at the outset if one considers the so-called argumentum, which is almost certainly itself a homily». Cette divergence de point de vue vient selon moi du fait que pour $\mathrm{P}$. Allen et W. Mayer, en première analyse et non sans raison, une série est forcément homogène et reflète un original, alors qu'il me semble qu'en deuxième analyse, une série est par définition «composée»: j'y reviens plus loin.

24. Telle est l'hypothèse, prenant appui sur M. Cunningham, Preaching and the Community, dans Church and People in Byzantium, éd. R. Morris, Birmingham 1990, p. 29-47, que formu- 
taires continus (sur Isaïe et sur Job, ainsi que sur les Proverbes et sur l'Ecclésiaste) plaide en tout cas en faveur de l'authenticité des argumenta, sans permettre toutefois aucune certitude a priori.

Le troisième cas concerne la doxologie, soit qu'elle a été ajoutée à un écrit qu'on a voulu faire passer pour une homélie (la possibilité ne doit pas être a priori exclue), soit qu'elle a été modifiée, intentionnellement ou non, par un copiste. Dans certains cas, elle permet de deviner l'inauthenticité d'un texte. A. Wenger a ainsi remarqué que les homélies de Chrysostome se terminent huit ou neuf fois sur dix par la même finale, contenant tout ou partie de la for-

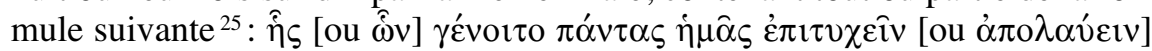

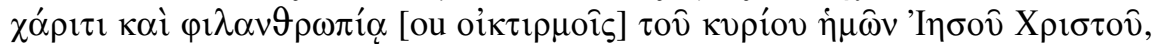

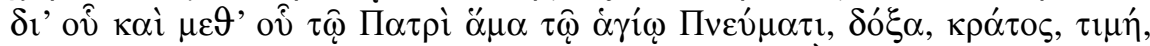

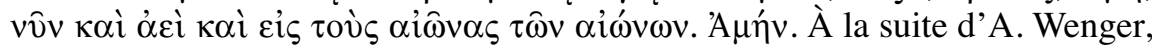
$\mathrm{S}$. Voicu a utilisé ce critère, parmi d'autres, pour montrer l'inauthenticité de plus d'un texte dont la doxologie était différente ${ }^{26}$. La fidélité de la tradition à cette doxologie réputée authentique est en tout cas un indice non négligeable pour l'unité oratoire et éditoriale de l'œuvre chrysostomienne.

De façon plus générale, le cas des argumenta et celui des doxologies illustrent la possibilité, attestée par ailleurs ${ }^{27}$, que des textes écrits aient été composés par l'auteur ou travestis, du vivant de l'auteur ou non, de façon à leur donner l'apparence d'homélies réellement prononcées. Ils confirment dans le même temps la possibilité, inverse, d'un regroupement de plusieurs homélies dans un ensemble littéraire écrit. À quoi il faut ajouter la possibilité intermédiaire, à savoir que des textes comme les Explications des Psaumes aient constitué «pour le prédicateur (y compris avec l'aide de tachygraphes) un matériau préparatoire à des homélies, susceptible au besoin de développements circonstanciés ${ }^{28}$.

lent P. Allen et W. Mayer dans deux de leurs articles: Chrysostom and the preaching of homilies in series: A new approach to the twelve homilies In epistulam ad Colossenses (CPG 4433), $O C P 60,1994$, p. 22; Chrysostom and the preaching of homilies in series: A re-examination of the fifteen homilies In epistulam ad Philippenses (CPG 4432), cité n. 22, p. 278.

25. Jean Chrysostome. Huit catéchèses baptismales (SC 50 bis), Paris 1970, p. 57-59.

26. Voir, par exemple, La volontà e il caso: la tipologia dei primi spuri di Crisostomo, dans Giovanni Crisostomo. Oriente e Occidente tra IV e v secolo. XXXIII Incontro di studiosi dell'antichità cristiana (Roma, 6-8 maggio 2004), I, Rome 2005, p. 101-118, ici p. 102; L'immagine di Crisostomo negli spuri, dans Chrysostomosbilder in 1600 Jahren. Facetten der Wirkungsgeschichte eines Kirchenvaters (Arbeiten zur Kirchengeschichte 105), éd. R. BRÄNDLE et M. WallRafF, Berlin-New York 2008, p. 61-96, spéc. p. 64 et 72.

27. P. Allen et W. Mayer, Chrysostom and the preaching of homilies in series: A new approach to the twelve homilies In epistulam ad Colossenses (CPG 4433), cité n. 24, p. 21-22, citent avec raison le cas d'Augustin, qui, selon son biographe Possidius de Calame, aurait ajouté à ses 119 Enarrationes in Psalmos 86 textes qu'il aurait dictés en forme d'homélies: Possidius, Librorum omnium et tractatuum uel epistolarum sancti Augustini episcopi indiculum, X, 4, éd. A. Wilmart, Miscellanea Agostiniana, II, Rome 1931, p. 181-182.

28. R. C. Hill, St. John Chrysostom. Commentary on the Psalms, I, Brookline MA 1998, p. 16. Le problème se pose de façon plus aiguë encore pour certains commentaires continus, comme celui sur Job: voir H. Sorlin, Jean Chrysostome. Commentaire sur Job (SC 346), I, Paris 1988,p. 35 n. 1. 
Le problème de loin le plus complexe est celui des révisions et des recensions multiples, comme pour les homélies sur Jean ${ }^{29}$, sur les Actes ${ }^{30}$ ou sur Tite et Philémon ${ }^{31}$. Plusieurs cas de figure sont envisageables :

1. Chrysostome lui-même a retravaillé ses textes. C'est l'hypothèse la plus optimiste, mais nous n'en avons pas de preuve explicite.

2. Des «éditions pirates» ont pu être diffusées avant la mise en circulation par l'auteur d'un texte achevé par ses soins. Mais là encore, pas de preuve explicite.

3. Pirates ou non, plusieurs notes sténographiques des mêmes homélies ont pu être prises en même temps ${ }^{32}$, aboutissant à plusieurs versions; les notes ellesmêmes devant faire l'objet d'une interprétation par le calligraphe chargé de produire une copie lisible, les mêmes notes ont pu occasionner deux transcriptions différentes. Le cas des homélies sur l'Épître aux Hébreux, transcrites après sa mort par Constantinos, prêtre d'Antioche, est un exemple célèbre de ce genre de transcription ${ }^{33}$, mais il est un peu isolé, et il reste à confronter au fait que la série est hétérogène et artificielle ${ }^{34}$. Cette transcription n'en est pas moins un passage obligé qui a son importance, comme à la fin de ce qu'il reste du Commentaire sur Isaïe, où, après le ch. 8, v. 10, le grec s'arrête sur

29. Voir P. W. Harkins, The Text Tradition of Chrysostom's Commentary on John, Studia Patristica, VII, 1966, p. 210-220; M.-É. BoISMARD et A. LAmouille, déjà cités, Un évangile préjohannique, 3 vol., 6 tomes (Études bibliques 17/18, 24/25, 28/29), Paris 1993/1994/1996.

30. Voir E. R. Smothers, Le texte des homélies de saint Jean Chrysostome sur les Actes des Apôtres, Recherches de science religieuse 27, 1937, p. 513-548; A Problem of Text in Saint John Chrysostom, Recherches de science religieuse 39, 1951, p.416-442; Toward a Critical Text of the Homilies on Acts of St. John Chrysostom, Studia Patristica, I, 1957, p. 53-57; F. Gignac, Evidence for deliberate scribal revision in Chrysostom's Homilies on the Acts of the Apostles, dans Nova \& Vetera. Patristic Studies in Honor of Thomas Patrick Halton, éd. J. Petruccione, Washington DC 1998, p. 209-225.

31. B. Goodall, The Homilies of St. John Chrysostom on the Letters of St. Paul to Titus and Philemon. Prolegomena to an Edition, University of California Press, Berkeley-Los AngelesLondres 1979; W. FICK [PRAdELs], Les homélies de Jean Chrysostome sur les Épîtres à Tite et Philémon: texte grec, traduction et commentaire, thèse, Univ. de Strasbourg 2, 1992.

32. Cf. Socrate, Histoire ecclésiastique, VI, iv, 9, éd. G. C. Hansen, P. Périchon et P. Maraval (SC 505), Paris 2006, p. 271-273, qui parle de plusieurs ỏ

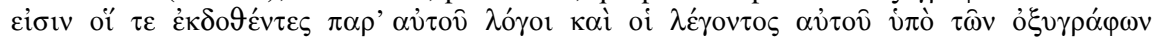

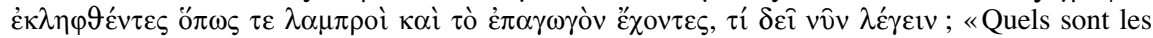
discours qu'il a produits et qui ont été enregistrés par les sténographes lorsqu'il parlait, à quel point ils sont admirables et persuasifs, pourquoi faudrait-il le dire maintenant?» Cf. aussi le témoignage, peu fiable, du Ps.-Georges D'AlexAndrie, Vie de saint Jean Chrysostome, 42, éd. F. Halkin, Douze récits byzantins sur saint Jean Chrysostome, Bruxelles 1977, p. 197: ' $\mathrm{Hv} \delta \dot{\varepsilon}$

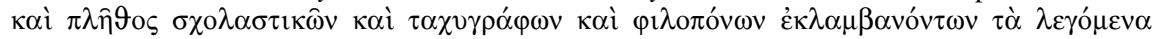

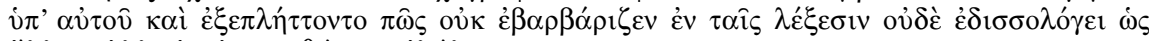

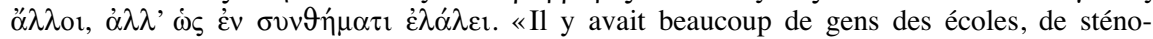
graphes et d'érudits qui prenaient en notes ce qu'il disait, et ils étaient frappés de voir comme son style était tout sauf barbare, combien il évitait de se répéter - comme d'autres le font - et à quel point il parlait de façon concise».

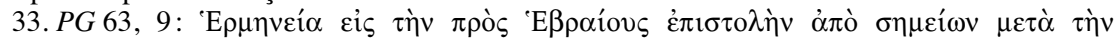

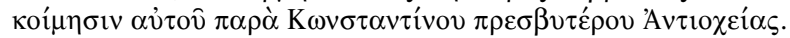

34. Cf. P. Allen et W. Mayer, The thirty-four homilies on Hebrews: the last series delivered by Chrysostom in Constantinople?, Byz. 65, 1995, p. 309-348. 
ces mots ${ }^{35}$ : «Jusqu'ici, c'est en caractères grecs qu'on trouve présentée cette introduction du bienheureux et très saint archevêque de Constantinople, Jean Chrysostome, mais à partir d'ici, c'est par des signes». Une indication que reproduit la version arménienne ${ }^{36}$, transmise dans un manuscrit du $12^{\mathrm{e}}$ siècle, avant de fournir un commentaire sur le reste du livre: le reste des signes tachygraphiques avait-il été déchiffré par un scribe plus compétent, ou bien le copiste arménien a-t-il ajouté une exégèse étrangère à Chrysostome?

4. Il y a eu deux «éditions», l'une à Antioche, l'autre à Constantinople; de fait, quelle que soit la nature de ces «éditions», la dispersion géographique est une réalité qui peut servir d'explication à une partie des disparités textuelles.

5. Des amis ou des partisans de Chrysostome ont plus ou moins massivement «publié» ses œuvres, au besoin en les remaniant. Dès avant sa mort des écrits pseudochrysostomiens ont commencé à circuler ${ }^{37}$.

6. Les «éditions» successives, dans les manuscrits, apportent à leur tour divers remaniements.

Quelles que soient les possibilités envisagées, il semble peu probable d'arriver à prouver leur réalité; et, en définitive, entre deux états textuels, c'est paradoxalement le moins abouti qui a le plus d'autorité ${ }^{38}$, car le moins suspect d'avoir été retouché ou «amélioré». Ce qui remet en question l'opinion communément partagée selon laquelle le style authentique de Chrysostome, tel que nous pouvons le lire dans le «texte uni » souvent reproduit dans les éditions modernes, est toujours poli et parfaitement élégant. La tradition estelle responsable de ce polissage? L'histoire des textes permet en tout cas de vérifier que, lorsqu'elle intervient, la tradition va généralement dans le sens de la simplification et de l'harmonisation, conformément à «l'horizon d'attente» de chaque époque. Et comme tout horizon, celui de notre époque est lui aussi limité.

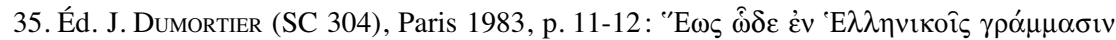

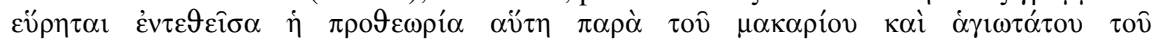

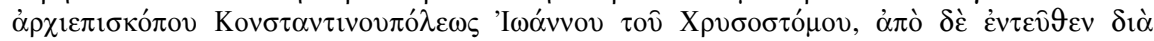

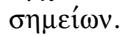

36. Ibidem, p. 13.

37. Sur le rôle des johannites dans la diffusion des chrysostomica et des pseudochrysostomica, voir S. J. Vorcu, «Furono chiamati giovanniti...»: Un'ipotesi sulla nascita del corpus pseudocrisostomico, dans Philomathestatos. Studies in Greek and Byzantine Texts Presented to Jacques Noret for His Sixty-Fifth Birthday, éd. B. Janssens, B. Roosen et P. Van Deun (Orientalia Lovaniensia Analecta 137), Louvain 2004, p. 701-711. Sur la typologie et les circonstances des pseudochrysostomica, voir notamment S. Vorcu, La volontà e il caso, cité n. 26, p. 101-116.

38. Cf. J. Quasten, Patrology, III, Utrecht-Anvers 1960, p.433, traduit par J. Laporte, Initiation aux Pères de l'Église, III, Paris 1962, p. 607-608. 


\section{2. - L'ENTOURAGE DU TEXTE}

En dehors du texte, ou plutôt autour de lui, la tradition manuscrite présente une série de marques qui toutes sont pertinentes pour l'histoire du texte. En effet, chaque manuscrit est en lui-même une édition pouvant comporter des éléments «péritextuels » à la fois sur l'ensemble du livre et sur chaque texte qu'il contient.

Concernant l'ensemble du codex peuvent être indiqués :

1. le titre de l'œuvre ou de la collection de textes, avec le nom de l'auteur ou des auteurs,

2. parfois, le $\pi i ́ v \alpha \xi$ ou index des textes, avec la numérotation des pièces.

Autour de chaque texte peuvent se trouver:

1. le titre du texte, au début et/ou à la fin, avec le nom de l'auteur et le numéro dans l'acolouthie du manuscrit ou de la série,

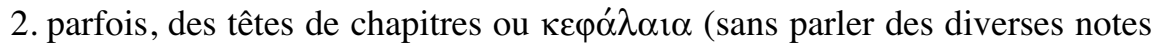
et gloses marginales),

3. souvent, la stichométrie,

4. généralement, la «signature» du copiste.

Je m'intéresserai ici aux seuls titres, notamment parce qu'ils fourmillent de renseignements sur le genre littéraire, le contexte historique ou liturgique, le lieu, le sujet ou le passage biblique commenté. Je prendrai comme exemple les Homélies sur les Statues, où un premier sondage dans les manuscrits montre que, comme souvent dans une tradition textuelle où l'accumulation prévaut sur l'élimination, les titres ont tendance à s'allonger avec le temps; car ils ne remontent certainement pas à l'auteur, et leur ancienneté est généralement douteuse.

Pour les Statues, le titre contient dans les manuscrits un ou plusieurs des 8 éléments suivants :

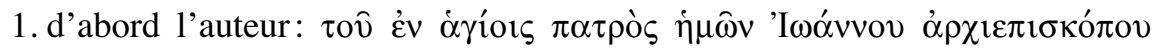

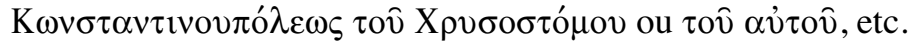

2. puis le genre littéraire général: $\dot{\alpha} \mu \imath \lambda \hat{i} \alpha(\imath)$

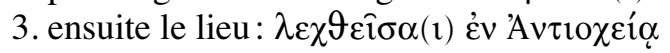

Ces trois premiers éléments sont les plus généraux; ils sont dans les manuscrits la vraie «signature» de la série. D'autres s'ajoutent et varient selon chaque homélie:

4. le contenu exégétique (cf. hom. 1: «sur la parole de l'apôtre: Usez d'un

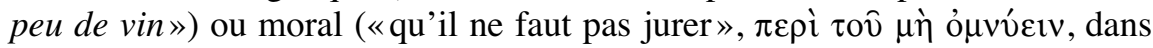
beaucoup d'homélies); c'est cet élément qui prend le plus d'ampleur avec le temps, jusqu'à devenir un résumé (à vrai dire très partial et partiel), énumé-

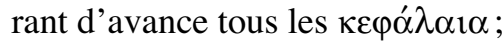

5. le ton ou le genre littéraire particulier d'une partie au moins du texte:

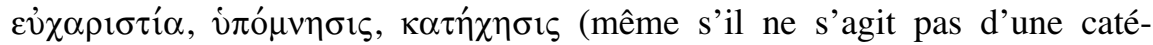

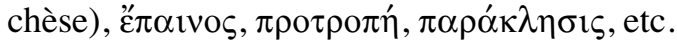

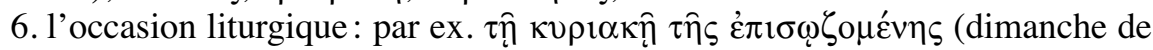

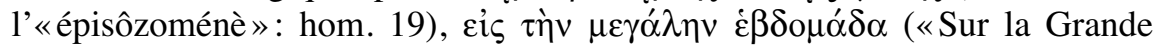
Semaine» de Pâques: In Ps. 145, homélie jointe à la série dans certains manuscrits) 


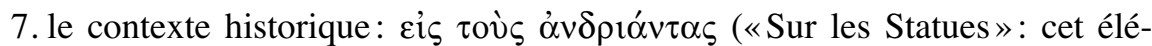
ment de titre n'est pas général dans les manuscrits, ce n'est qu'un élément secondaire et occasionnel)

8. le numéro dans la série (c'est souvent le dernier élément du titre): ó $\mu \imath \lambda i ́ \alpha$ $\alpha^{\prime}, \dot{o} \mu \imath \lambda i^{\prime} \alpha \beta^{\prime}$, etc.

Ce qu'il y a de plus significatif peut-être, c'est que le titre des homélies est le lieu par excellence où une série est rendue visible en tant que telle. La question se pose de façon aiguë en ce qui concerne les Statues, et elle touche à une responsabilité majeure de la tradition vis-à-vis de l'œuvre chrysostomienne: les regroupements en série.

\section{3. - LES SÉRIES DE TEXTES}

Inspirant directement les éditions modernes, la tradition manuscrite présente des séries d'homélies groupées d'après des critères thématiques, chronologiques, exégétiques ou liturgiques. Or P. Allen et W. Mayer ont montré qu'au sein de certaines séries, des homélies peuvent provenir aussi bien de Constantinople que d'Antioche ${ }^{39}$. Ce manque d'unité et cette hétérogénéité de date ou de lieu ne peuvent que trahir leur caractère secondaire.

Le cas le plus flagrant et le plus connu est celui des 12 homélies dites Contre les anoméens, prononcées en partie à Antioche en 386-387, en partie à Constantinople en 398. En réalité, cette série artificiellement constituée par Montfaucon, en englobe une autre, très bien attestée dans les manuscrits, intitulée Sur l'incompréhensibilité de Dieu et comportant 8 pièces, les 5 premières publiées en SC 28 bis, ainsi que trois autres homélies, la $11^{\mathrm{e}}$ Contre les anoméens (CPG 4324), Le Fils est consubstantiel au Père (CPG 4320) et Sur la demande de la mère des fils de Zébédée (CPG 4321), publiées par A.-M. Malingrey en SC 396, sous le titre Sur l'égalité du Père et du Fils ${ }^{40}$. Parmi les nombreux autres exemples qui pourraient être cités, W. Mayer a montré pour sa part que la troisième des Homélies sur le changement des noms est à séparer de la séquence que les deux premières forment à la suite des quatre homélies Sur le commencement des Actes et avant le Sermon 9 sur la Genèse, luimême suivi par l'Homélie 4 sur le changement des noms ${ }^{41}$.

39. En plus des études sur les homélies sur l'Épître aux Colossiens, sur l'Épître aux Philippiens et sur l'Épître aux Hébreux déjà citées, mentionnons, de W. MAYER seule, 'Les homélies de s. Jean Chrysostome en juillet 399'. A second look at Pargoire's sequence and the chronology of the Novae homiliae (CPG 4441), BS 60/2, 1999, p. 273-303; The Homilies of St John Chrysostom: Provenance. Reshaping the foundations (OCA 273), Rome 2005; Les homélies de Jean Chrysostome: problèmes concernant la provenance, l'ordre et la datation, Revue des études Augustiniennes et Patristiques 52, 2006, p. 327-351; The sequence and provenance of John Chrysostom's homilies In illud: Si esurierit inimicus (CPG 4375), De mutatione nominum (CPG 4372) and In principium Actorum (CPG 4371), Augustinianum 46, 2006, p. 169-186.

40. Ces trois homélies sont respectivement dans la série de Montfaucon contre les anoméens les homélies 11, 7 et 8. Voir aussi A.-M. Malingrey, Prolégomènes à une édition des homélies de Jean Chrysostome, Contra Anomoeos, Studia Patristica, XXII, 1989, p. 154-158.

41. Les homélies de Jean Chrysostome, cité n. 39, p. 347-348. 
Il me semble donc nécessaire de réfléchir sur ce qu'est une série ${ }^{42}$, en prenant comme exemple ce qu'on pourrait appeler une «série factice», celle sur les Statues. La définition de la série pourrait reposer sur trois critères combinés :

1. Le témoignage des manuscrits. Une homélie est une performance orale et événementielle, et non d'abord un texte ou un fait littéraire. Par conséquent, une série d'homélies n'est pas forcément le fidèle reflet d'une chronologie, mais c'est avant tout un fait éditorial et un fait de tradition, attesté par les manuscrits ou les éditions modernes; en tant que tel, il est indépendant de l'auteur. La responsabilité de l'auteur n'est donc pas engagée (mais son autorité l'est bien!) dans la mesure où, comme ici, il n'y a pas de sa part de projet éditorial connu. Il devient dès lors très délicat de vouloir reconstituer une série «originale» (la tradition manuscrite peut se fonder sur une réalité historique, ou non), mais c'est aussi un avantage: une édition dans les Sources Chrétiennes ne vise pas forcément à reconstituer cet hypothétique original, mais d'abord, par sa nature même, à se placer au sein d'une tradition éditoriale. En tout état de cause, malgré les risques, très grands pour Chrysostome, d'artificialité et même d'inauthenticité, le premier critère pour la définition de la série est le témoignage des manuscrits - mais il n'est pas le seul.

2. En deuxième lieu intervient le cadre chronologique, à la fois géographique et historique (la crise des Statues) et liturgique (le carême), de février à avril 387.

3. En troisième lieu importe le contenu ou, comme ici, le projet pastoral (et non pas éditorial) de l'auteur, qui est d'abord un pasteur. En l'occurrence, le projet de Chrysostome pour le carême 387 est de débarrasser les fidèles d'Antioche de l'habitude de jurer: c'est un fil directeur très net pour la série des Statues.

F. Van de Paverd a bien remarqué le «désordre» logique des homélies dans la séquence transmise par les manuscrits ${ }^{43}$; le nombre total d'homélies est d'ailleurs variable, de 19 à 24 pour les témoins les plus complets ${ }^{44}$. Le caractère «factice» de l'ensemble se laisse donc deviner, mais il me semble tout bonnement inutile d'employer ce qualificatif à partir du moment où toute série, étant avant tout un fait de tradition, est une fabrication éditoriale; je préfère donc employer le terme de série «composée».

Là est manifestement l'apport le plus décisif de la tradition à l'œuvre de Chrysostome: en présentant des séries de textes, elle leur confère un statut et une forme littéraires. En un mot, la tradition contribue à faire des homélies et

42. Je ne fais qu'esquisser ici une réponse à la question soulevée avec justesse par P. ALLEN et W. MaYer, Chrysostom and the preaching of homilies in series: A new approach to the twelve homilies In epistulam ad Colossenses (CPG 4433), cité n. 24, p. 21: «Indeed, in Chrysostom's regard, what constitutes a homiletic series is a problematical question».

43. F. Van de Paverd, St. John Chrysostom, the Homilies on the Statues. An Introduction (OCA 239), Rome 1991, p. Xxvi-Xxx et 205-362.

44. Voir A. Valevicius, Les 24 homélies De statuis de Jean Chrysostome. Recherches nouvelles, Revue des Études Augustiniennes 46, 2000, p. 83-89. 
des écrits de Jean Chrysostome des œuvres à part entière. L'auteur a bien «publié» de son vivant quelques-uns de ses textes: le Dialogue sur le sacerdoce, mentionné par Jérôme en 392, son traité Sur la virginité ${ }^{45}$, les traités épistolaires confiés à Olympias ${ }^{46}$ ou adressés à Théodore, à Stagire, à Stéléchius, à Démétrius, et d'autres encore ${ }^{47}$. Mais pour l'ensemble, le fait est difficile à prouver. S. Voicu estime avec raison que Chrysostome ne se souciait guère de la mise en circulation de ses écrits ${ }^{48}$. La tradition manuscrite et les éditions imprimées après elle ont donc une grande responsabilité, celle de produire en tant qu'œuvre littéraire cohérente et identifiable des performances orales prises à la volée, des morceaux de prédication et des textes qui autrement seraient peut-être restés isolés. Paradoxalement, la tradition garantit à Chrysostome son propre statut d'auteur. La présentation ou la fabrication des séries est donc loin d'être insignifiante !

\section{4. - LES TEXTES DÉRIVÉS}

C'est un domaine où la tradition a été le plus directement active et créative: le Chrysostome authentique a inspiré divers résumés, adaptations et

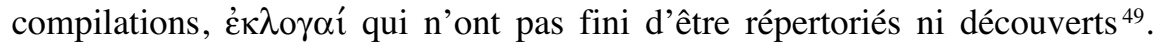

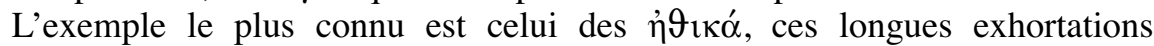
morales qui forment en général la dernière partie des homélies exégétiques ${ }^{50}$

45. Voir ce qu'il en dit à la fin de l'homélie 19 sur la Première épître aux Corinthiens,

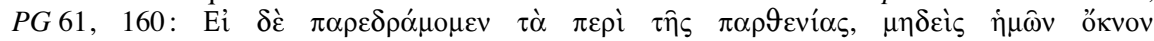

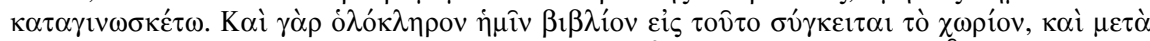

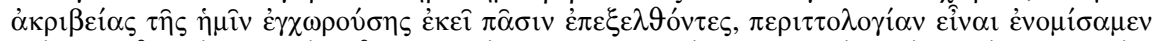

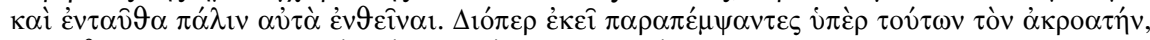

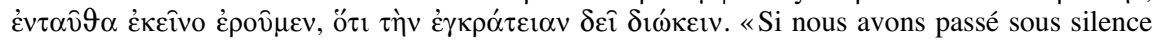
ce qu'il y a à dire sur la virginité, que personne ne nous accuse de négligence. Car nous avons composé un livre entier sur ce passage; et après y avoir traité ce sujet avec autant de soin qu'il nous a été possible, nous avons cru inutile d'y revenir aujourd'hui. Nous y renvoyons donc notre auditeur, et nous nous contentons de dire ici qu'il faut garder la continence».

46. Quod nemo laeditur nisi a seipso = Jean Chrysostome. Lettre d'exil, éd. A.M. Malingrey (SC 103), Paris 1964; Ad eos qui scandalizati sunt ob aduersitates = Jean Chrysostome. Sur la Providence, éd. A.-M. Malingrey (SC 79), Paris 1964. La lettre XVII, 4c à Olympias (SC 13 bis, p. 384) mentionne le premier, tout en étant elle-même jointe au second.

47. Par exemple hom. 88, 4 sur Matthieu, $P G 58,779-780$ : «Je préférerais ne pas ressentir un besoin pressant de vous adresser ce conseil, mais plutôt parler de la bataille contre les juifs, les païens, les hérétiques... Dans d'autres livres se trouvent de nombreux discours que nous avons prononcés sur ces sujets, cependant nous ne pouvons pas célébrer la victoire totale dans

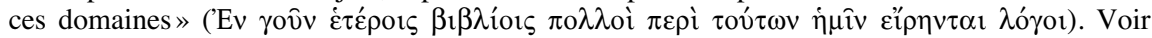
entre autres A. Olivar, La predicación cristiana antigua, Barcelone 1991, p. 930, et surtout H. Lietzmann, Johannes Chrysostomos, RE (Pauly-Wissowa), IX, 1916, c. 1817, qui pense qu'il s'agit des homélies Contre les anoméens, Contre les Juifs et De s. Babyla contra Iulianum et Gentiles.

48. S. J. VoIcu, Pseudo-Giovanni Crisostomo: i confini del corpus, Jahrbuch für Antike und Christentum 39, 1996, p. 107.

49. Un appendice spécifique est réservé dans chaque volume des Codices Chrysostomici Graeci à la description des «nouveaux textes» répertoriés.

50. Voir, par exemple, ce qu'en dit, de façon très significative, B. DE MontFaucon dans le Monitum ad commentarium in epistolam ad Galatas, reproduit en $P G 61,609$ (je traduis): 
et qui ont été parfois isolées du reste des homélies, puis rassemblées et transmises en collections indépendantes. Un autre exemple est celui des chaînes exégétiques, dont le succès même, attesté pour Chrysostome jusque dans l'Occident latin, a manifestement compromis la survie de plus d'un commentaire en tradition directe ${ }^{51}$. C'est là un domaine en soi et, par prudence, je ne m'y attarderai pas, car, à l'évidence, nous sortons là du texte dans son statut primaire et original.

\section{Conclusion}

En définitive, la transmission de l'œuvre de Jean Chrysostome me semble symptomatique du rapport qu'il y a entre tradition (avec un petit «t») et Tradition (avec un grand « $T »$ ): non seulement dans leurs significations respectives, l'une purement historique et contingente, l'autre théologique et pérenne, mais aussi dans une perspective littéraire et épistémologique, au sens où la tradition manuscrite informe ou infirme une autre tradition, celle qu'on pourrait appeler la tradition de connaissance, élaborée et transmise par les érudits et les théologiens au sujet de Chrysostome et des Pères de l'Église en général. En d'autres termes, tradition textuelle et tradition «intellectuelle» entrent en interaction. En effet, comme tous les Pères, Jean Chrysostome est à la fois objet et sujet de tradition: ses écrits sont objets de transmission, mais son œuvre est elle-même une partie de la Tradition de l'Église qu'elle a contribué à former. Or il est évident qu'il y a un lien entre la tradition manuscrite et la tradition ecclésiale ou intellectuelle. Car si l'on en vient à constater, comme je viens de le faire, qu'il n'y a pas seulement dans la tradition textuelle une transmission fidèle, mais une nécessaire et massive transformation de l'original, les conséquences sur la nature ou du moins sur certains aspects de la tradition intellectuelle en découlent immédiatement.

La première conclusion à en tirer est la nécessité d'une «distinction sans séparation » entre tradition textuelle et tradition intellectuelle. Il est significatif de constater que, pour la première fois dans l'histoire, il y a avec la Patrologie de Migne un textus receptus des Pères, et ce, bien au-delà de l'Église romaine catholique. Oui, la Patrologie est bel et bien devenue tradition,

\footnotetext{
«Dans ce commentaire, Chrysostome n'a pas procédé selon son habitude. De fait, dans ses autres commentaires du Nouveau Testament, tout est divisé ou distribué en sermons ou homélies; de sorte que le sermon se clôt sur une exhortation morale et que ces enseignements finaux d'ordre moral forment souvent la majeure partie de l'homélie. Or dans ce commentaire, tout est disposé autrement. En effet, de façon continue, Chrysostome y expliquait jusqu'à la fin chacun des versets de l'épître: il y adjoint parfois des enseignements moraux lorsque l'occasion s'y prête, mais ne clôt pas le sermon comme à son habitude. Et il n'est pas rare qu'il s'adresse à l'assemblée. C'est pourquoi je serais tenté de croire qu'il a fait ainsi : soit il a [d'emblée] lu ou récité ses textes en présence de l'assemblée, soit, après avoir divisé son commentaire en plusieurs parties et y avoir ajouté après coup un épilogue éthique concluant chaque partie, il a fait comme à son habitude et a prononcé devant l'assemblée son œuvre distribuée en homélies. Quant à savoir laquelle des deux méthodes il a employée, nous ne le pouvons sans divination».

51. Les commentaires sur les Proverbes ou sur Jérémie en sont de bons exemples.
} 
dans le même mouvement par lequel elle achève (aux deux sens du terme) toute tradition. Devant une telle réussite, qui dépasse aussi bien la rivalité cordiale d'un Fronton et d'un Savile que les visées apologétiques pourtant très ambitieuses de l'abbé Jacques-Paul Migne, plus d'un aura cédé à la tentation d'assimiler la Tradition des Pères à ce texte imprimé largement insatisfaisant. Une tentation doublement dangereuse, car elle rend traditionnel, du point de vue textuel aussi bien qu'intellectuel, un état du texte qui, d'une part, n'est pas forcément représentatif de la tradition manuscrite et qui, d'autre part, infléchit la compréhension de la Tradition jusque dans le détail des passages invoqués par les théologiens et les historiens modernes. L'absence de distance critique est bien sûr tout aussi dommageable ici que ne l'est ou ne le serait la séparation de la tradition intellectuelle vis-à-vis des questions de transmissions textuelles, comme si elle se trouvait dans un isolement fixiste, désormais hors du temps. Prise à la lettre, la Patrologie est un monument parfait pour le dogmatisme comme pour le positivisme historique; elle ne doit pas faire oublier qu'il n'y aura jamais d'édition ni de critique définitives.

La deuxième remarque que je voudrais faire, c'est que nous sommes en présence d'un cercle herméneutique, dans lequel les œuvres de Jean Chrysostome, à l'instar des Écritures, intègrent leur propre réception. Par conséquent, dans le motif qu'ils forment ensemble, les fils du tissu textuel se fondent et ne permettent plus de distinguer l'original du secondaire: Chrysostome est à ce point porté par la tradition qu'il ne fait plus qu'un avec elle. La critique textuelle joue-t-elle dès lors ici le rôle subversif qu'on a pu lui prêter face à la Révélation chrétienne? La déconstruction méthodique des connaissances supposées oblige en réalité, nous l'avons vu, à revenir à une tradition plus authentique, où finalement la tradition est connue et reconnue comme telle. Dans cette herméneutique chrysostomienne qui est aussi notre épistémologie, la grande différence avec ce que disent les théologiens concernant les Écritures est que nous ne pouvons pallier la perte de l'authenticité originelle par l'autorité spirituelle et l'action continue de l'Esprit Saint à travers les siècles. Éditeurs, traducteurs, commentateurs, tous orphelins de Chrysostome, nous sommes condamnés à assumer notre propre part de tradition, et même de paternité, sur une œuvre qui est la sienne et aussi un peu la nôtre.

Guillaume BADY

Université de Lyon, UMR 5189 HiSoMa (CNRS/Université Lumière-Lyon 2) Institut des Sources Chrétiennes 